\title{
PEDIATRICS \\ Repeated botulinum toxin-A injections safe in children
}

Botulinum toxin-A (BTX-A) injections into the detrusor are an effective treatment for neurogenic bladder dysfunction in adults and children. Although the longterm safety of such injections has been confirmed in adults, data in the pediatric population are lacking. Preliminary results from a new study published in The Journal of Urology show that repeated BTX-A injections are safe in children with neurogenic bladder dysfunction and do not induce additional fibrosis in the bladder wall.

As fibrosis is a major finding in samples of neurogenic bladder wall, Pascali and colleagues wanted to determine whether one or more BTX-A injections in children would cause histopathological changes to the this tissue. The researchers enrolled 40 children (aged 2-18 years, both males and females) with neurogenic bladder dysfunction who had failed to respond to conventional first-line treatment (anticholinergic agents and clean intermittent catheterization).

Patients received $10 \mathrm{IU} / \mathrm{kg}$ (maximum dose 300 IU) BTX-A into the detrusor, either once or multiple times. Participants were followed up every 3 months, for up to a year after treatment, to assess urodynamic changes, continence status and presence of urinary tract infections. Furthermore, bladder wall biopsies were carried out before and after treatment. The samples were then examined for histological changes and categorized according to the levels of infiltration of inflammatory cells, edema, and fibrosis.

Overall, 46 bladder wall biopsy samples were obtained. Some degree of histological alteration was observed in all samples. No differences in inflammation or edema were observed between specimens collected prior to and after BTX-A treatment. Repeat BTX-A injections did not induce increased

\section{4 ...there seemed to be decreased fibrosis in biopsy samples after multiple treatments 77}

fibrosis in the bladder wall-in fact, there seemed to be decreased fibrosis in biopsy samples after multiple treatments $(P<0.5)$.

"Our data suggest that in the pediatric population intradetrusor BTX-A injections in the bladder wall do not increase fibrosis," write the study authors. They acknowledge that their results, although encouraging, should be considered preliminary owing to some limitations of the study. The uncontrolled design, small patient population and limited follow-up reinforce the need for further research to confirm the long-term effects of BTX-A injections in children.

\section{Katrina Ray}

Original article Pascali, M. P. et al. Effects of botulinum toxin type $A$ in the bladder wall of children with neurogenic bladder dysfunction: a comparison of histological features before and after injections. J. Urol. doi:10.1016/j.juro.2011.01.019 